\title{
The perception of medical students and trainees about a career as a cardiothoracic surgery
}

\author{
Ariana Axiaq ${ }^{1}$, Raneesha Pillay ${ }^{2}$, Manasi Shirke$^{1}$, Sara Zaidi $^{3}$, and Amer Harky ${ }^{4}$ \\ ${ }^{1}$ Queen's University Belfast \\ ${ }^{2}$ Queen Mary University of London Barts and The London School of Medicine and \\ Dentistry \\ ${ }^{3}$ King's College London \\ ${ }^{4}$ Liverpool Heart and Chest Hospital NHS Foundation Trust
}

February 16, 2021

\begin{abstract}
Background: Cardiothoracic (CT) surgery is a dynamic and demanding specialty, which is popular amongst medical students thus, posing as a favourable career choice for many. However, there is a significant proportion of medical students who prefer to choose other specialities instead, for different reasons. Aim of the study: This review aims to identify factors affecting the uptake of cardiothoracic surgery as a career by medical students, junior doctors and trainees globally. Methods: A comprehensive literature review was conducted using PubMed, EMBASE, SCOPUS and CINAHL using specific keywords including "cardiothoracic surgery" AND "medical student" AND "career". Inclusion and exclusion criteria were also developed to ensure only relevant studies were used for the paper. Information on the perspectives, knowledge, and beliefs on cardiothoracic surgery amongst medical students and trainee doctors worldwide was collected. Results: Most data was sourced from UK and US-based studies with only a minority of literature from other parts of the world. Uptake of cardiothoracic surgery amongst medical students, junior doctors and trainees is generally low, on a global level. Deterring factors identified from this review included work-life balance, professional satisfaction, lifestyle, and family planning, the latter being especially important for female medical students. Conclusion: Although job posts are still being filled, the increasing numbers of medical students losing interest in a career in cardiothoracic surgery needs to be addressed. Areas of future research into this area would be to re-assess medical school curricula and opportunities to engage more in the field whilst at medical school and beyond.
\end{abstract}

\section{The perception of medical students and trainees about a career as a cardiothoracic surgery}

Ariana Axiaq ${ }^{1}$, Raneesha Pillay ${ }^{2}$, Manasi Mahesh Shirke ${ }^{1}$, Sara Zaidi ${ }^{3}$, Amer Harky MRCS, MSc ${ }^{4,5,6}$

Running Head: cardiothoracic surgery as a career

1. School of Medicine, Queen's University Belfast, Belfast, UK

2. Barts and The London, School of Medicine and Dentistry, London, UK

3. King's College London, London, UK

4. Liverpool Centre for Cardiovascular Science, University of Liverpool, Liverpool, UK

5. Department of Cardiothoracic Surgery, Liverpool Heart and Chest Hospital, Liverpool, UK

6. Liverpool Centre for Cardiovascular Science, University of Liverpool and Liverpool Heart and Chest Hospital, Liverpool, UK

\section{Corresponding Author}

Amer Harky 
MSc, MRCS

Department of Cardiothoracic Surgery

Liverpool Heart and Chest Hospital, Liverpool

e-mail: aaharky@gmail.com

tel: $+44-151-600-1616$

Funding: No funding was obtained

Conflict of Interest: All authors have no conflict of interests to declare

\begin{abstract}
Background: Cardiothoracic (CT) surgery is a dynamic and demanding specialty, which is popular amongst medical students thus, posing as a favourable career choice for many. However, there is a significant proportion of medical students who prefer to choose other specialities instead, for different reasons. Aim of the study: This review aims to identify factors affecting the uptake of cardiothoracic surgery as a career by medical students, junior doctors and trainees globally.
\end{abstract}

Methods: A comprehensive literature review was conducted using PubMed, EMBASE, SCOPUS and CINAHL using specific keywords including "cardiothoracic surgery" AND "medical student" AND "career". Inclusion and exclusion criteria were also developed to ensure only relevant studies were used for the paper. Information on the perspectives, knowledge, and beliefs on cardiothoracic surgery amongst medical students and trainee doctors worldwide was collected.

Results: Most data was sourced from UK and US-based studies with only a minority of literature from other parts of the world. Uptake of cardiothoracic surgery amongst medical students, junior doctors and trainees is generally low, on a global level. Deterring factors identified from this review included work-life balance, professional satisfaction, lifestyle, and family planning, the latter being especially important for female medical students. Conclusion: Although job posts are still being filled, the increasing numbers of medical students losing interest in a career in cardiothoracic surgery needs to be addressed. Areas of future research into this area would be to re-assess medical school curricula and opportunities to engage more in the field whilst at medical school and beyond.

Key Words: perception, interest, career, cardiothoracic surgery, medical students, trainees

\title{
Abbreviations and acronyms
}

Cardiothoracic - CT

\section{Introduction}

Over the past decade, applications and interest in the surgical training pathway have plummeted, eventually affecting cardiac surgical specialties as well. Analysing data from the United States, a gradual decline in the physician to patient ratio has been documented with lesser applications being made to the CT surgery training pathway, despite the medical school seats remaining fairly constant (1). Trends in applications for thoracic fellowships in comparison to other subspecialties from 1997 to 2012 showed a decline of more than $50 \%$ of applicants in the training pathway (2). These statistics prove to be worrisome due to the aging population of the current CT surgeons and increasing demand for the declining workforce. This in turn places more pressure to attract a greater number of students to train in cardiothoracic surgery (3).

A 2005 survey by Brundage et al. found that $45 \%$ of the first-year students were interested in a surgical career but only $7 \%$ were matched to surgical residencies. Reasons attributed to this perceived 'loss of interest' were the length of training, residency lifestyle, hours, call schedule, and female gender of the student respondent. Although lifestyle issues remained at the forefront of student concerns, those who initially portrayed interest 
particularly liked the intellectual challenge, career opportunities, financial rewards, and technical skills that came along with a surgical career (4).

Aforementioned, unlike any other surgical specialty, CT surgery has also been hit with decreased numbers of applicants. With concerns about a shortage of CT surgeons in the upcoming decade, several studies have been conducted to ascertain the reasons behind this regression. Potentially, strong candidates lose their interest in a career in CT surgery during medical school and hence do not apply for the training pathways. Algethami et al. highlighted the need for an adequate introduction to the field of cardiothoracic surgery, emphasised the need to increase exposure and to provide close mentorship in order to entice a wide array of students to pursue a career in cardiothoracic surgery (5). Moreover, only $15.8 \%$ of United Kingdom medical graduates who were interested in cardiothoracic surgery, chose to pursue further specialized training. Likewise, in the United States, graduating medical students are showing a greater preference for other surgical specialties compared to cardiothoracics (4).

In order to combat this perceived loss of interest, several strategies have been suggested to target medical students early on in their careers. Moreover, early exposure, positive, experiences, inspiring role models, and career insight are key features to be taken into account while making a specialty choice as a medical student or junior doctor. Previously, the method of preferentially selecting high-achieving, resilient, and hardworking individuals early on in their careers and encouraging them to seek CT surgery was implemented (6). Currently, an increase in interest in the field has been documented by the application of stimulation-based technology to augment skills and interest (7). Increasingly, summer research programs and scholarships have shown to increase interest among medical students $(8,9)$. The interest of students keen on CT surgery as a career must be maintained throughout medical school by means of appropriate mentorship. Additionally, CT surgery must be implemented as a part of the undergraduate curriculum in medical schools in the UK and worldwide. This would improve student experience and help develop their interest (10).

An understanding of the factors that attract or deter medical students, junior doctors, and trainees to a career in CT surgery must be acquired in order to increase applications to the training pathway. Hence, the primary objective of this review is to scope the level of interest of medical students, junior doctors, and trainees in cardiothoracic surgery. Furthermore, we aim to gain further understanding of the factors that pertain to students developing or losing interest in the field.

\section{Material and Methods}

\subsection{Search Strategy}

This review was conducted in accordance with the PRISMA guidelines. To facilitate the literature search, selection criteria in terms of keywords and inclusion/exclusion criteria were implemented. A comprehensive literature search was conducted on PubMed, EMBASE, SCOPUS, and CINAHL. Keywords included were "cardiothoracic surgery" AND "medical student" AND "career". A staged literature search was performed, and relevant articles are cited and referenced in the paper.

\subsection{Inclusion and exclusion criteria}

For the purpose of this review, studies concerning medical students, junior doctors, and surgical trainees were included. Whereas, those documenting non-healthcare professionals or fully-fledged CT specialists were excluded. Published randomised controlled trials, observational studies, and interventional studies investigating the knowledge, interest, skills, and attitudes in CT surgery were available for inclusion. Conference papers and editorials, literati studies with less than 20 participants, studies with only CT consultants, fellows, or CT trainees, and those not in the English language were excluded from this review. Studies that did not address our primary or secondary objectives were excluded as shown in Figure 1.

\subsection{Quality assessment}

The quality of each publication was evaluated independently by four authors. A full-text review and data collection from all studies meeting our inclusion criteria was performed independently by the authors and 
any disagreement was reached by consensus. Additionally, data were extracted and validated by all authors independently using the Rayyan QCRI software.

\subsection{Data extraction}

All relevant articles were identified and screened; the results are summarized in a narrative manner within the text of this review, with a summary table provided where appropriate. It was not possible to conduct an appropriate statistical analysis due to the limited research data among the studies on this subject.

\section{Results}

\subsection{Characteristics of the studies:}

\subsubsection{Location, aim, and population}

The results from a total of 20 articles were analysed in this review. Of these, 12 papers were from the US, 5 from the UK, and 1 from Sudan, Nigeria, and Saudi Arabia each. The population of these studies consisted of medical students, foundation doctors, core surgical trainees, interns, and general surgery residents. Any papers that included only $\mathrm{CT}$ trainees, professors or consultants were excluded from this review. These papers discussed the level of interest, perceptions on a career in CT surgery, factors that impacted decision making and interventions used in medical education such as simulation, surgical anatomy lessons, scholarship awards, and mentorship to expose students and trainees to CT surgery at an earlier stage. A couple of studies also discussed the barriers in which female medical students may need to overcome and the factors that motivate their career choice in CT. The length of training, the presence of female role-models in CT surgery, and lifestyle were key factors involved in decision-making.

\subsubsection{Perceptions about cardiothoracic surgery}

Whilst many studies attempted to quantify interest amongst preclinical and clinical medical students, junior doctors, and even core surgical trainees, it is difficult to ascertain if such interest will ultimately result in CT surgery being the final choice of surgery.

With only 3 included studies in Asia or Africa, the trends observed in these studies may not reflect the overall international student perspective of medical students about the specialty. However, in Alawad et al. and Algethami et al., the proportion of interest is comparatively low, being $16.50 \%$ (11) and 4.50\% (5) respectively. Another important result is the overall discrepancy between male and female interest in the specialty, particularly at a preclinical level; men were keener and more involved in the specialty as students.

\subsubsection{Factors considered in pursuing a career in cardiothoracic surgery}

A number of papers evaluated the various factors that shape perception and interest of CT surgery as a career. In Algethami et al. (5), 17 factors were identified, out of which 5 were considered attractive or strongly attractive and 2 categorised as deterring factors. The most attractive quality was the ability to save and influence patients' lives (65.9\%) followed by the skills involved in surgery (55.3\%) and the flexibility of practicing worldwide $(59.2 \%)$. The main challenging factors identified were the limited geographical locations to practice in Saudi Arabia as well as the competitive nature of jobs and training posts. In a similar study by Preece R. et al. (12), the same discouraging factors resulted to be the most significant however, in addition to the encouraging factors reported in Algethami et al., intellectual challenge (72.4\%) and the opportunity to do research and innovate (69.0\%) were also key factors. In the UK study, more students regarded the ability to practice worldwide as an important factor (79.3\% compared to $59.2 \%$ ).

Coyan et al. (13) compared the determinants of CT surgery as the top choice in interested students and non-interested students. For both groups, work/life balance was the highest-ranked deterrent factor ( $82 \%$ of responses) followed by other factors such as limited pathology of patients, length and difficulty of training, high stress and constantly dealing with death and personalities of CT surgeons. Meanwhile, personal satisfaction and intellectual challenge were highly regarded equally . However, for students with interest, research opportunities, and potential for new technologies were more highly valued whilst work-life balance was not 
as highly influential. Dissimilarly in non-interested students: Professional satisfaction and work-life balance were both important. In women, other crucial points were highlighted such as the ability to address health inequalities as a positive factor and the impact of work of family planning as a negative quality. Similar findings were reported in Meza et al. (14). Despite intellectual interest (91\%) and lifestyle (86\%) being the main goals influencing the choice of career, the ability to address health disparities ( $52 \%$ responses) and salary $(46 \%)$ were not uncommonly noted (Figure 2 ).

\subsubsection{Exposure}

Several studies to support the hypothesis of early exposure and its impact on a career in CT surgery have been investigated with the earliest included in this review being Chen H. et al. (15). Students who had ambitions to subspecialise in CT and other surgical specialties saw significantly more procedures in their respective subspecialty interests. This study concluded that while students had equal exposure to general surgery due to medical school curricula requirements, their exposure to other surgical specialties including CT surgery correlated highly with their interest in the respective fields. From the years 2003-2012, 30 US medical students in a single institution participated in a program designed to expose students to basic surgical skills, clinical operations, and developing research projects with a cardiac surgeon. Of the 23 who applied for residency, 12 (52.2\%) matched into general surgery while 2 matched into CT surgery. (14)

In Algethami M.R. et al. (5), $74.6 \%$ of final year students and $88.3 \%$ of students felt they were not sufficiently exposed to CT surgery. Only $7.8 \%$ of the students were assigned mentors where $24 \%$ of them managed to obtain contact details from $\mathrm{CT}$ surgeons for future research purposes and $8.4 \%$ attended conferences or careers day. $56.4 \%$ of those without mentors revealed they had no interest in CT surgery and $70.4 \%$ were unaware of the current CT training pathway. The impact exposure has on career choice was further explored in other studies in the UK. In Gasparini M. et al. (16), $71 \%$ had some CT exposure whilst $24.7 \%$ had extracurricular exposure. Confidence in career choice in CT increased by an average of $20.4 \%$ ( $\mathrm{p}=0.0244)$ from 5.76/10 to 7.24/10 after exposure. Preece R. et al. (12) showed that $75 \%$ of the cohort reported a lack of exposure within the curricula and only $13 \%$ had extracurricular exposure via conferences or careers day. Burnside M. et al. (17) concluded that of the 20 trainees that had CT jobs, $75 \%$ indicated returning to CT surgery while trainees that had no CT surgery jobs chose not to return to the specialty either as a 1st or $2^{\text {nd }}$ preference.

\subsection{Interventions to Facilitate Exposure}

\subsubsection{Simulations}

A few studies have been done on investigating different interventions in medical education to foster an early interest in CT surgery. In Macfie R. et al. (23), 31 US medical students, 26 general surgery residents, and $15 \mathrm{CT}$ fellows participated in 7 tissue-based simulations supervised by 6 CT faculty in a single institution from 2015-2017. The pre and post-surveys revealed that CT simulation participation significantly increased operative confidence and interest across all training levels. Of the 44 that were not confident pre-simulation, $75 \%$ increased their confidence levels by 1 or more with $39 \%$ of them experiencing an increase in 2 or more levels. The magnitude of increase in interest levels was significantly larger earlier in training (43.06 for medical students, 29.17 for PGY1-4, and 27.78 PGY5 to fellow level at $\mathrm{p}=0.001$ ).

A recent evaluation by Coyan G. et al. (19) on simulation-based electives, showed an increase in those who scrubbed in or observed CT cases from $9 \%$ pre-course to $33.3 \%$ post-course $(\mathrm{p}=0.11)$. In addition to that, the ease of acquiring a mentor significantly increased from $23.8 \%$ pre-course to $66.7 \%$ post-course $(\mathrm{P}=0.01) .81 \%$ reported that the mini-elective significantly increased their CT knowledge when compared to the standard curriculum. A similar 2010 study involving 44 medical students at a US institution also showed that post-course, $61 \%(\mathrm{p}=0.001)$ agreed that their interest increased significantly. In terms of subspecialty selection, $18 \%$ chose thoracic surgery pre-course versus the $39 \%$ post-course. This also proved to be a good experience amongst female participants as only $3(12 \%)$ selected a thoracic rotation pre-course but $9(35 \%)$ selected it post-course (24). 


\subsubsection{Workshops Student-Led Led Extracurricular Activities}

A 2015 analysis of a pre and post-intervention survey with a $92.6 \%$ response rate. The 50 responses showed that the understanding of the CT training pathway and academic pathway increased from $20 \%$ to $80 \%$ $(\mathrm{p}<0.001)$ and $20 \%$ to $70 \%(\mathrm{p}<0.001)$ respectively. Furthermore, $77 \%$ strongly agreed that the event increased their interest in CT surgery (25). Similarly, in George J. et.al.(26), 80 UK medical students completed a questionnaire after attending a workshop comprising of operative videos, career seminars, basic surgical skills, and animal heart dissections. The survey showed an increase in CT surgery interest by $42 \%$ as a direct result of the workshops.

\subsubsection{Surgical Anatomy Curriculum}

A 2009 study was conducted amongst 211 first-year US medical students where, of those who volunteered, 25 were randomly selected to undergo a surgical anatomy curriculum throughout the year. The remaining cohort underwent the standard anatomy curriculum. The post-evaluation questionnaire showed that the positive opinion of surgeons before and after the program improved greatly $(\mathrm{p}<0.008)$ as compared to the overall cohort $(\mathrm{p}<0.003)$ and that $13 / 25(52 \%)$ felt that their grades improved. However, there was no significant difference in the final anatomy grades post-evaluation when compared to the rest of the cohort (27).

\subsubsection{Impact of Scholarship Awards}

Trehan $\mathrm{K}$ et al. described a review of the long-term interest of 45 scholarship recipients for research and clinical activities in CT surgery. These recipients were evaluated prospectively using a US institutional database and showed that $17.4 \%$ of these students went on to choose CT surgery (9).

\section{Discussion}

CT surgery is a relatively small specialty however, recruitment into the field has been declining $(28,1)$. In the UK, only $18 \%$ of newly qualified CT surgeons and $32 \%$ of current trainees are from UK medical schools. In the US whilst CT surgery residency posts are always filled, the number of unmatched applicants has been decreasing. Given the inadequate research in other regions, whether this is applicable to medical students elsewhere is yet to be deduced however studies such as by Algethami et al. reveal similar trends.

\subsection{Interest in CT surgery as a career}

Multiple studies report medical students' perceptions about pursuing a career in CT surgery. Interest has been relatively low compared to other specialties such as general surgery despite the popularity of a surgical career. However, both interest in surgery and CT surgery seem to vary across stages of training, whether medical, premedical or at postgraduate clinical training level as well as gender.

Prospects of a CT surgery career were more highly regarded amongst preclinical students, in particular first year medicals students, in comparison to clinical year students. Even more so, interest is further diminished upon progression to foundation year, core surgical training (17) (in UK) or general surgical residency $(18,15)$ (in US). Besides, premedical students were found to be more enthusiastic about CT surgery than preclinical medical students (22). This may be due to loss of interest due to the lack of exposure in CT surgery throughout medical school and medical training owing to a lost opportunity to gain insight into the specialty. More students would have been involved in extra-curricular activities in other specialties and would be less likely to consider a specialty which they were not exposed to (17). In a cross-sectional study by Ghannam et al. (29), baseline interest in CT surgery was moderately high in high school students at $15 \%$ as compared to baseline interest in medicine and surgery at $38 \%$ and $24 \%$ respectively. Similarly, high baseline interest was reported in Sood et al. (22), raising a question about when the optimal time of exposure to the specialty would be to maximise and retain student interest.

\subsection{Factors influencing perception of a career in CT surgery}

The ability to actively influence patients' lives as well as intellectual challenge are predominant reasons 
supporting selection of CT surgery careers. Respondents with a declared interest in the specialty have different established interests than non-interested participants with the former allocating more value to research opportunities and new technological procedures (13). Nonetheless, various dissuading factors persist, mainly in concern to the difficulty and length of training and work-like balance (5) as well as the personality of CT surgeons $(20)$ and limited opportunities $(5,12)$. Interestingly, a history of previous scandals in the field was not influential on the perception of CT surgery as a prospective career. Such decision-making elements should all be considered in the design of exposure programmes and recruitment schemes in order to provide a realistic portrayal of the surgical area and encourage fruitful and inspiring interactions between trainees and consultants already in the specialty and more junior members.

The overall perspective of CT surgery relates to its surgical emergency-heavy nature, but it is not solely constituted of trauma and emergency patients. CT surgeon-orientated programmes should be focused on offering practice patterns of surgeons to better inform students on lifestyle. Enabling students to envision these challenges is essential for a balanced representation of the career without any concealment or misinformation (13). With core surgical trainees frequently holding a common interest in both CT surgery and vascular surgery, given the similar patient cases and vascular work, an exploration into the benefits of promoting these two specialties together may provide valuable insight (3).

Another noticeable discrepancy is the significant difference in interest between men and women, with men being more interested in CT surgery than women. In all studies and at all levels of study, interest in a CT career has been higher in men than in women. Despite the varying degree of this difference from one study to another, the extent of this disparity was generally reported to be lower in less advanced stages of training such as premedical and preclinical students, yet still evident just the same. Proposed explanations for this phenomenon include the difficulty to maintain a work-life balance especially in family planning as well as the difficulty of taking maternity leave during training or post-training and re-entering the specialty (21). Such deterring factors may be addressed through the introduction of more female cardiothoracic surgery mentors and family planning incentives such as less-than-full time training options (13).

\subsection{Levels of Exposure}

The articles in this review have showed that exposure at all stages of training influences perception, interest, and confidence in choosing CT surgery as a career choice. Diminished caseloads and the length of training programs (5) have also been indicated in to be a deterrent. The introduction of integrated programmes might shorten the duration of training, but it accompanies a set of perceived challenges surrounding the maturity and the vulnerability of attrition amongst trainees (30).

Lack of exposure in standard medical curricula amongst the institutions and countries involved in this review seems to be a recurring theme and any adequate exposure was largely obtained in an extracurricular environment via student selected components and mentorships organised by the students who developed an early interest in CT surgery. Increasing exposure to CT surgery throughout medical school and in junior surgical jobs, may help with maintaining long term interest. We can observe a pattern where students who intend to pursue a career in specific surgical fields including CT surgery tend to proactively organise theatre time as opposed to those who were not interested in CT surgery. However, due to the limited number of studies available, it may be difficult to discern if changing the current levels of exposure via designed programmes may affect the interest in CT surgery and thus acquiring an adequate workforce in the future.

\subsection{Interventions in Medical Education}

Although only a limited number of articles were published describing the effects of simulation $(19,23)$, conferences/careers day (25), workshops (26), and modified curriculum amongst medical students and trainees (27), they all showed that these interventions provided a means of networking and developing surgical skills. Students and junior doctors were immersed in a surgical environment and this influenced their decisions on career choice to a certain extent. Seminars and mentors provide a more realistic view on workload and lifestyle, again striving to remove students from the shielded environment of medical school as mentioned previously $(13,31)$. These simulations are a form of exposure. Therefore, the ability to determine the op- 
timal duration of the intervention and to track the results of surgical clerkships is vital in order to develop these programmes, as this will be the ultimate assessment of success.

Modifying existing anatomy curricula may not significantly affect the results during examination but it improved students' opinions of surgeons (27) and may instill surgical interest amongst medical students at an earlier stage. Indeed, this may result in a more holistic environment in medical education where students are exposed to both medical and surgical environments from their first year which perhaps may lead to changing perceptions of surgery and ultimately a career in niche fields like CT surgery.

Incentives such as scholarship awards provide the required opportunities to get involved in CT surgeryrelated research and inevitably maintain interest in the field over long periods of time as students have the benefit of gaining a mentor, whilst working together (9).

There will be challenges in implementing such changes in current medical school curricula due to the difficulties in making any improvements from feedback obtained by a small class and identifying the feasibility of involving a larger number of students in workshops and simulations. This is reflected in the studies exploring these interventions as their sample sizes were small $(\mathrm{n}<100)$. This could potentially be addressed by allowing students to rotate between the different surgical workshops throughout the year in small groups as they would in placement blocks. Incorporating surgical teaching in every clinical year in medical school may also allow for more opportunities in witnessing clinical cases such as CT surgery procedures and taking part in any organised surgical simulation or seminars regarding CT surgery.

\subsection{Mentorships}

Exposure to CT surgery whether in an extracurricular environment, within the medical school, or via the aforementioned methods of intervention, enhances the probability of networking and obtaining a mentor in CT surgery. Mentorships are pivotal in maintaining interest, providing technical training and career counselling amongst current CT trainees. The tailoring of mentorships to individual trainee needs may also be useful in improving career experience (32).

An analysis of the national training surveys on the GMC website from 2012 to 2019 indicated that whilst there was little variation on overall trainee satisfaction across the UK, trainees were least satisfied with teaching $(62.63 \%)$, rota design $(61.91 \%)$, and workload $(45.07 \%)$; the only area that had less than $60 \%$ satisfaction (10). Successful mentorships offering all-rounded support could have a positive effect on career satisfaction amongst CT trainees.

\section{Future research}

The research generated on the subject thus far is to be commended however, more study on global trends in medical student career choice and respective decision-making is opportunistic. This will offer variations in perceptions of interest, differences in barriers in immersing in a career in CT surgery, and efficiency of exposure programmes offered internationally.

Primarily, the barriers to medical students pursuing a career in CT surgery should be better understood in order to prioritise addressing challenges such as the length of training pathway, burnout rate, and opportunity of progression. With CT surgery becoming increasingly specialised as related fields like interventional cardiology expand, the perceived specialty versatility by medical students is to be assessed. This calls for more qualitative studies to truly assess the nature and significance of deterrents.

With mentoring and simulation programmes as the most favoured approaches to stimulate interest, better assessment is required on the effectiveness of these programmes in terms of method of delivery, taught content, type of interaction, duration, and best-suited audience. For example, longer follow-ups to analyse the proportion of participants enrolling in CT surgery training is suggested. Besides, medical students tend to change their career interests from preclinical years to clinical years. For this reason, the type of programme whether simulation, mentorship, or clinical apprenticeship as well as the time of exposure are to be evaluated (13). Further research on existing mentorship programmes could be done to produce clearly 
defined goals to ensure that everyone gains the most from their mentors in every aspect including academic and emotional support (32). Other forms of incentives such as research experiences should be included in the discussion. It should also be determined whether to target these programmes at already interested students or students with no declared interest. Another key point in increasing the attractiveness of the specialty is accessibility, the inclusion of more female CT surgeons in mentorships along with widening access schemes is recommended. The impact of same-sex mentorships or the presence of successful female CT surgeons on influencing their attitudes towards a CT career could be researched to affect change in female interest levels in a CT career. With the rising prominence of social media, it can potentially be used as a tool to build a network of same-sex mentors (33) and comparative studies could be done between obtaining mentorships via a scheme in medical school/trainee years and social media.

Furthering research about current student perspectives on CT surgery and career promotion strategies may only be as beneficial if supported with actionable incentives and guidelines for both medical education and career progression within the field.

\section{Conclusion}

From a global perspective, interest in pursuing a career in CT surgery amongst medical students is generally low. Although there are stark differences between countries, with UK and US medical students having relatively more interest than those in other parts of the world, most studies have demonstrated interest of less than 50\%. The key factors which deter medical students from pursuing a career in this field are work-life balance, professional satisfaction, and family planning, especially in female students. Salary and lifestyle factors are also important variables that affect students' perspectives.. Perhaps more methods of student involvement should be developed to provide a deeper insight into this field with workshops or other educational talks to help students address these deterring factors and create ways to manage them.

\section{References}

1. Salazar JD, Ermis P, Laudito A, et al. Cardiothoracic surgery resident education: update on resident recruitment and job placement. Ann Thorac Surg 2006;82:1160-5.

2. Pousatis SM, Marshall MB. Trends in applications for thoracic fellowship in comparison with other subspecialties. Ann Thorac Surg 2014;97:624-32; discussion 632-3.

3. Grover A, Gorman K, Dall TM, et al. Shortage of cardiothoracic surgeons is likely by 2020. Circulation 2009;120:488-94.

4. Brundage SI, Lucci A, Miller CC, Azizzadeh A, Spain DA, Kozar RA. Potential targets to encourage a surgical career. J Am Coll Surg. 2005 Jun;200(6):946-53. DOI: 10.1016/j.jamcollsurg.2005.02.033. PMID: 15922210. 5. Algethami MR, Bandah ST, Safhi MA, et al. Factors Influencing Saudi Medical Student's Decision Towards Cardiothoracic Surgery as a Future Career, a Cross-Sectional Study. Mater Sociomed. 2019;31(3):197-201. doi:10.5455/msm.2019.31.197-201 6. Peel JK, Schlachta CM, Alkhamesi NA. A systematic review of the factors affecting the choice of surgery as a career. Can J Surg. 2018;61(1):58-67. doi:10.1503/cjs.008217 7. Tesche LJ, Feins RH, Dedmon MM, et al. Simulation experience enhance medical students' interest in cardiothoracic surgery. Ann Thorac Surg 2010;90:1967-73; discussion 1973-4. 8. Haggerty KA, Beaty CA, George TJ, et al. Increased exposure improves recruitment: early results of a program designed to attract medical students into surgical careers. Ann Thorac Surg 2014;97:2111-4; discussion 2114. 9. Trehan K, Zhou X, Yang SC. Is medical student interest in cardiothoracic surgery maintained after receiving scholarship awards? Ann Thorac Surg (Internet). 2015;100(3):926-31. Available from: http://dx.doi.org/10.1016/j.athoracsur.2015.03.012 10. Chan J, Sidhu P. HOW MUCH CARDIOTHORACIC SURGERY IS TAUGHT IN UK MEDICAL SCHOOLS?. Ulster Med J. 2019;88(2):128. 11. Alawad AA, Khan WS, Abdelrazig YM, Elzain YI, Khalil HO, Elsayed OB, Adam OA. Surgery as a career choice among medical students at University of Medical Sciences and Technology. Sudan Med J. 2014 Apr;50(1):26-31. doi: 10.12816/0015568. 12. Preece R, Ben-David E, Rasul S, Yatham S. Are we losing future talent? A national survey of UK medical student interest and perceptions of cardiothoracic surgery. Interact Cardiovasc Thorac Surg. 2018 Oct 1;27(4):525-529. doi: 10.1093/icvts/ivy119. 13. Coyan GN, 
Kilic A, Gleason TG, Schuchert MJ, Luketich JD, Okusanya O, Kinnunen A, Sultan I. Medical student perceptions of a career in cardiothoracic surgery: Results of an institutional survey. J Thorac Cardiovasc Surg. 2020 May;159(5):1906-1912. doi: 10.1016/j.jtcvs.2019.07.022. 14. Meza, J.; Sood, V.; Proctor, E et al. Female medical student interest in cardiothoracic surgery: Barriers we must overcome. Journal of Surgical Research. 2013 Feb; 179 (2). 15. Chen H, Hardacre JM, Martin C, Lillemoe KD. Do medical school surgical rotations influence subspecialty choice? J Surg Res. 2001 May 15;97(2):172-8. doi: 10.1006/jsre.2001.6135 16. Gasparini M, Jayakumar S, Ayton S, Nardini MN, Dunning JD. Medical student exposure to cardiothoracic surgery in the United Kingdom. Interact Cardiovasc Thorac Surg. 2019 Mar 15:ivz038. doi: 10.1093/icvts/ivz038. 17. Burnside N. The impact of junior surgical jobs in cardiothoracic surgery on career choice in the United Kingdom. Gen Thorac Cardiovasc Surg. 2018 Jul;66(7):411-414. doi: 10.1007/s11748-018-0925-y. 18. Abioye IA, Ibrahim NA, Odesanya MO, Wright KO. The future of trauma care in a developing country: interest of medical students and interns in surgery and surgical specialties. Int J Surg. 2012;10(4):209-12. doi: 10.1016/j.ijsu.2012.03.003. 19. Coyan GN, D'Angelo MP, Kilic A, Gleason TG, Luketich JD, Aranda-Michel E, Okusanya O, Schuchert MJ, Kinnunen A, Sultan I. Evaluation of a simulation-based mini-elective on medical student interest in cardiac surgery. J Card Surg. 2019 Oct;34(10):901-907. doi: 10.1111/jocs.14143. 20. Davis TA, Yesantharao PS, Yang SC. Where Do We Begin? Building Blocks to Establish a Cardiothoracic Surgery Interest Group. Ann Thorac Surg. 2019 Jun;107(6):1854-1859. doi: 10.1016/j.athoracsur.2018.12.040. 21. Foote DC, Meza JM, Sood V, Reddy RM. Assessment of Female Medical Students' Interest in Careers in Cardiothoracic Surgery. J Surg Educ. 2017 Sep-Oct;74(5):811-819. doi: 10.1016/j.jsurg.2017.02.013. 22. Sood V, Reddy RM. An analysis of preclinical students' perceptions of cardiothoracic surgical procedures. Ann Thorac Surg. 2012 Sep;94(3):800-5; discussion 805-6. doi: 10.1016/j.athoracsur.2012.03.113. 23. Macfie R, Walcott-Sapp S, Watson J, Haley C, Dewey E, Schipper P. Association Between Simulation Curriculum and Learners' Confidence and Interest in Cardiothoracic Surgery. JAMA Surgery. 2018;153(10):968. 24. Tesche L, Feins R, Dedmon M, Newton K, Egan T, Haithcock B et al. Simulation Experience Enhances Medical Students' Interest in Cardiothoracic Surgery. The Annals of Thoracic Surgery. 2010;90(6):1967-1974. 25. Bridgeman A, Findlay R, Devnani A, Lim D, Loganathan K, McElnay P et al. Inspiring the next generation of Cardiothoracic Surgeons: an easily reproducible, sustainable event increases UK undergraduate interest in the specialty. Interactive CardioVascular and Thoracic Surgery. 2015;22(1):106-108. 26. George J, Combellack T, Lopez-Marco A, Aslam U, Ahmed Y, Nanjaiah P et al. Winning Hearts and Minds: Inspiring Medical Students into Cardiothoracic Surgery Through Highly Interactive Workshops. Journal of Surgical Education. 2017;74(2):372-376. 27. Haubert L, Jones K, Moffatt-Bruce S. Surgical Clinical Correlates in Anatomy: Design and implementation of a first-year medical school program. Anatomical Sciences Education. 2009;2(6):265-272. 28. Westaby S, Baig K, De Silva R, Unsworth-White J, Pepper J. Recruitment to UK cardiothoracic surgery in the era of public outcome reporting. Eur J Cardiothorac Surg 2015;47:679-83 29. Ghannam M, Zhao L, Reddy RM. Current interest in careers in surgery and cardiothoracic surgery from the millennial generation. J Surg Educ. 2014 Sep-Oct;71(5):668-73. doi: 10.1016/j.jsurg.2014.02.004. 30. Lebastchi A, Tackett J, Argenziano M, Calhoon J, Gasparri M, Halkos M et al. First nationwide survey of US integrated 6-year cardiothoracic surgical residency program directors. The Journal of Thoracic and Cardiovascular Surgery. 2014;148(2):408-415.e1. 31. Chen H, Reddy R, Palmer S, Coleman D, Hoch J, DiMusto P et al. Attrition rates in integrated vascular and cardiothoracic surgery residency and fellowship programs. Journal of Vascular Surgery. 2019;69(1):236-241. 32. Stephens E, Goldstone A, Fiedler A, Vardas P, Pattakos G, Lou X et al. Appraisal of mentorship in cardiothoracic surgery training. The Journal of Thoracic and Cardiovascular Surgery. 2018;156(6):2216-2223. 33. Luc J, Stamp N, Antonoff M. Social Media as a Means of Networking and Mentorship: Role for Women in Cardiothoracic Surgery. Seminars in Thoracic and Cardiovascular Surgery. 2018;30(4):487-495. 


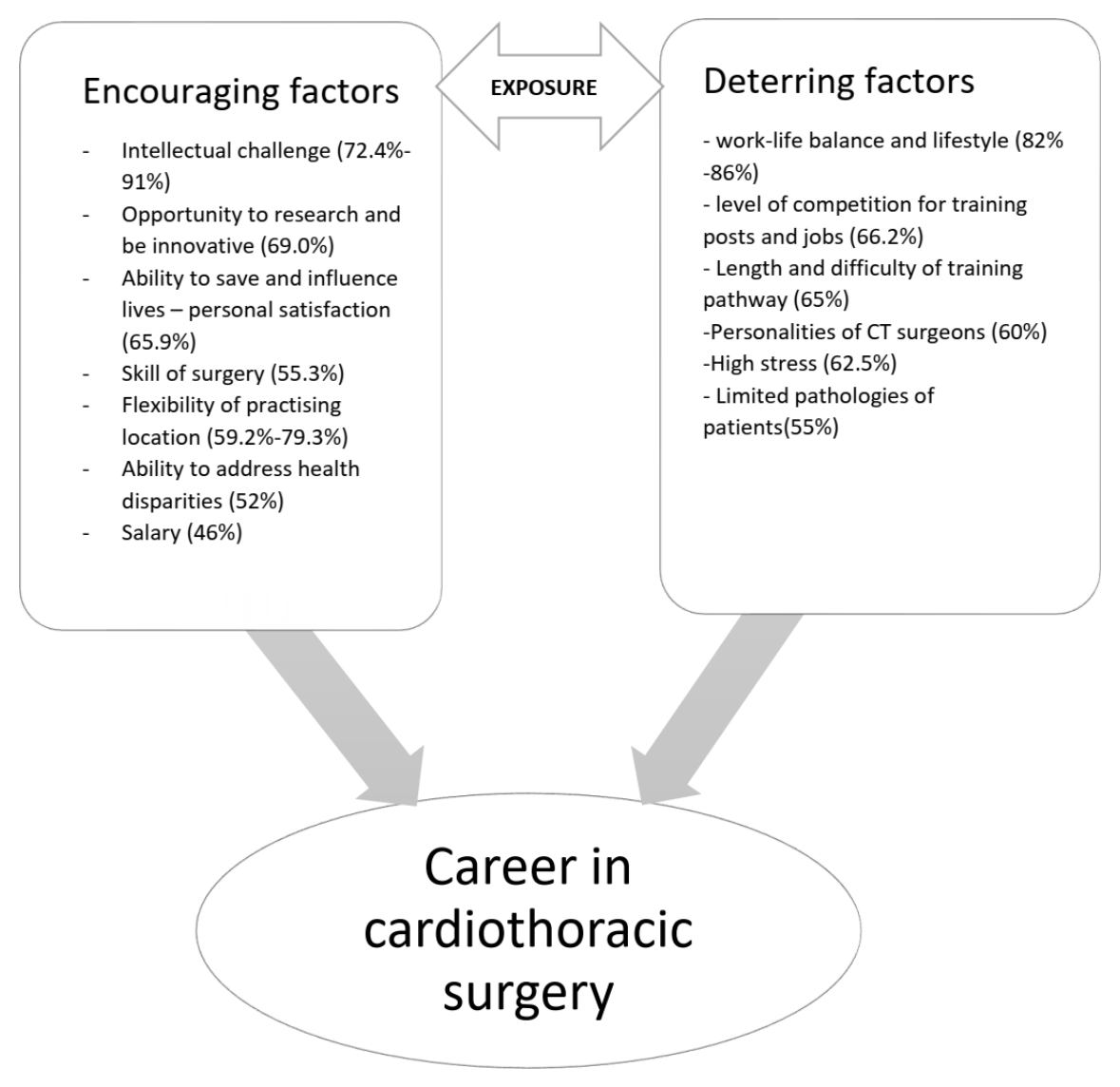



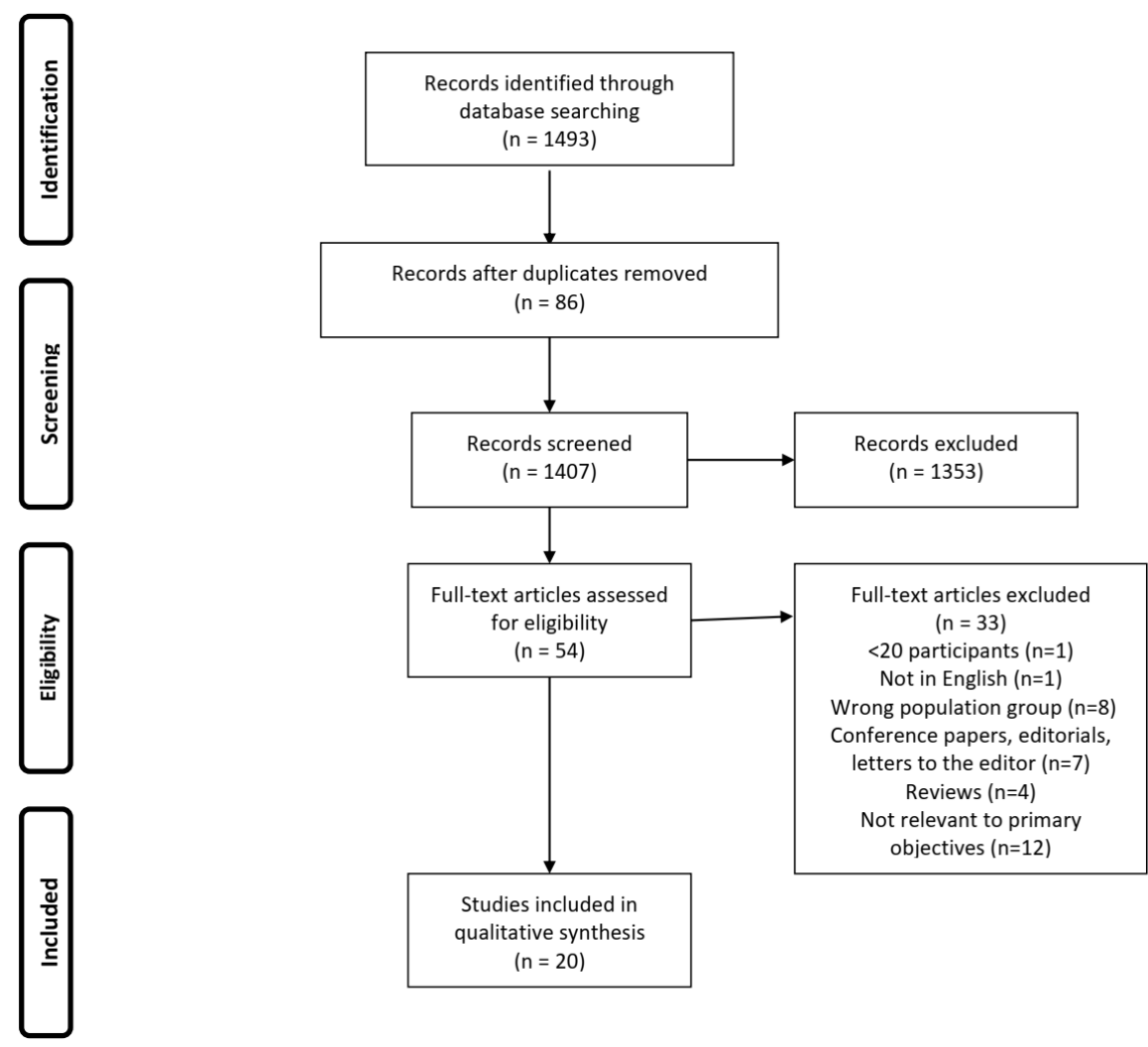

Studies included in qualitative synthesis $(n=20)$ 